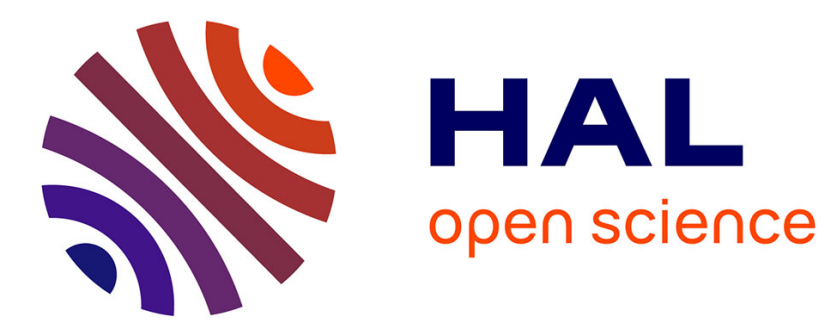

\title{
Monitoring silver solid-state dewetting with in situ ellipsometry
}

P. Jacquet, M. Kildemo, J. Teisseire, I. Gozhyk, J. Jupille, R. Lazzari

\section{To cite this version:}

P. Jacquet, M. Kildemo, J. Teisseire, I. Gozhyk, J. Jupille, et al.. Monitoring silver solid-state dewetting with in situ ellipsometry. Applied Surface Science, 2016, 421 (Part B), pp.553-556. 10.1016/j.apsusc.2016.10.160 . hal-01401507

\section{HAL Id: hal-01401507 https://hal.sorbonne-universite.fr/hal-01401507}

Submitted on 23 Nov 2016

HAL is a multi-disciplinary open access archive for the deposit and dissemination of scientific research documents, whether they are published or not. The documents may come from teaching and research institutions in France or abroad, or from public or private research centers.
L'archive ouverte pluridisciplinaire HAL, est destinée au dépôt et à la diffusion de documents scientifiques de niveau recherche, publiés ou non, émanant des établissements d'enseignement et de recherche français ou étrangers, des laboratoires publics ou privés. 


\title{
Monitoring silver solid-state dewetting with in situ ellipsometry
}

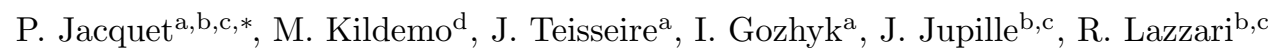 \\ ${ }^{a}$ Surface du Verre et Interfaces, UMR 125 CNRS/Saint-Gobain Recherche, 39 quai Lucien Lefranc, F-93303 Aubervilliers, France \\ ${ }^{b}$ CNRS, UMR 7588, Institut des NanoSciences de Paris, 4 place Jussieu, F-75252 Paris, France \\ ${ }^{c}$ Sorbonne Universités, UPMC Univ Paris 06, UMR 7588, Institut des NanoSciences de Paris, 4 place Jussieu, F-75252 Paris, France \\ ${ }^{d}$ Norwegian University of Natural Science and Technology (NTNU), 7491 Trondheim, Norway
}

\begin{abstract}
We consider the possibility of monitoring silver solid-state dewetting with in situ Ellipsometry. We first study the optical response of partially dewetted samples in correlation with their morphological structure measured by Atomic Force Microscopy (AFM). We find that the main features observed in microscopy are identifiable in Ellipsometry spectra. Then, we analyse the dielectric function extracted from in situ measurements to predict the progression in the dewetting process. We also identify two different behaviours leading to different final states.
\end{abstract}

Keywords: Solid-state dewetting, ellispometry, in-situ, thin films

Silver films deposited on a silica substrate do not wet, but are unstable and tend to agglomerate [1, 2]. This phe- 35 nomenon occurs below the melting point of the metal and is called solid-state dewetting. It has mainly been consid5 ered as a source of defects in silver-based insulated glazings produced by glass industry, and thus as an unwanted and detrimental phenomenon. However, it was recognized re- 40 cently that it is also a promising method to obtain, through templated dewetting, silver nanostructures having poten10 tially strong interests [3, 4. For instance, silver nano-grids can act as transparent electrodes; silver nano-particles can enhance photovoltaic devices efficiency through light trap- 45 ping [3]. Each application requires a good control of the silver structure and morphology. As a consequence, the fun15 damental understanding of metallic film dewetting gained more and more importance in the past years. Recently, in situ and real-time techniques have been proven to be a 50 powerful tool for studying this phenomenon [5, 6, 7]. These recent works are mostly based on in situ electronic micro20 scopies (either transmission (TEM) or scanning (SEM)) which are invaluable to understand dewetting kinetics and mechanisms at local scale. However, these methods have 55 inherent drawbacks: samples are restricted in size, can only be studied in a dedicated chamber and electron beam 25 possibly affects the dynamics of the phenomenon. The goal of the present work is to monitor dewetting in operating conditions without interfering. Since ellipsometry 60 is a photon-based technique and has a reasonable acquisition time (a few seconds for the full spectrum), it is a so candidate of choice. Moreover, silver is a test bed for optics because of its intense plasmonics response and poorly damped Drude electron behaviour 8 . Ellipsometry or related techniques (i.e. reflectivity) were

${ }^{*}$ Corresponding author intensively used to study silver nano-structures. To cite a few, Oates et al. dealt with silver deposition [9, nanoparticles (NP) [10] and more generally silver plasmonic response [11]. Silver deposition is of interest since it is the counterpart to dewetting: it is a transition between silver isolated particles and a continuous film. It was studied during either sputtering [9, 12, or physical vapour deposition [12, 13, 14, 15. In these studies, the plasmonic resonance of silver played a great part into the identification of the state of the layer (particle or continuous) as well as morphology evolution and characteristic lengths.

In the present study, our previous knowledge of dewetting morphology [7] is combined with temperature dependent ellipsometric measurements. Silver dewetting involves three distinct steps of morphology evolution that we aimed to pinpoint with ellipsometry: induction, propagation and sintering. During induction, there is no change in substrate coverage, but an overall roughening due to grain growth [7] with eventually the first hole appearance. Then, during propagation, holes grow and the substrate coverage decreases. Some grains are selected to grow before becoming isolated particles. At the end of propagation, the remaining particles sinter and tend slowly to agglomerate further into round-shaped particles close to equilibrium shape.

$20 \mathrm{~nm}$ thick silver film were deposited by sputtering (Ar pressure: $8.10^{-3}$ mbar, power: $210 \mathrm{~W}$, rate: $1.3 \mathrm{~nm} / \mathrm{s}$ ) onto (100)-oriented silicon wafers with one-side polished and covered with their native oxides. Wafers were $300 \mu \mathrm{m}$ thick for post mortem studies and $800 \mu \mathrm{m}$ thick for in situ ellipsometry to avoid bending while heating. Silver films were elaborated in the same batch, thus ensuring that the deposition conditions are rigorously identical for all of them. In the following, the only difference in sam- 
ple preparation for post-mortem or in situ studies was the wafer thickness. Post mortem samples were annealed in tific Instruments). To obtain different stages of dewetting, the different samples were annealed at 100, 150, 300 and $400^{\circ} \mathrm{C}$ with an heating ramp of $150^{\circ} \mathrm{C} / \mathrm{min}$; the temperature was maintained for $5 \mathrm{~min}$ before cooling. Atomic

75 Force Microscopy imaging (AFM) was performed ex situ post mortem on cooled samples with an AFM Dimension Icon (Bruker) apparatus.

In situ and real time measurements were performed using the heating stage adapted to the dual rotating compensator (RC2)-spectroscopic ellipsometer (provided by J.A. Woollam Co.). High quality data were recorded with online geometrical sample adjustments to correct for misalignments due to sample bowing, induced by heating. Reasonable acquisition times for full spectra (0.7-5.9 eV

${ }_{85}$ in 2-10 seconds) were obtained using the InGaAs and $\mathrm{Si}$ spectrographs on the detector arm. Spectra were recorded 125 without interruption during the experiments. The extraction of the film dielectric function $\left(\varepsilon=\varepsilon_{1}+i \varepsilon_{2}\right)$ and its analysis was performed with the CompleteEase software 90 and is described below.

Post mortem ellipsometry measurements, as well as the ${ }^{130}$ AFM pictures, were performed at room temperature after annealing at different temperatures $\left(100,150,300,400^{\circ} \mathrm{C}\right)$. Fig. 1 shows the sequence of AFM pictures. The first im-

95 age $(\mathrm{A})$ represents the layer as deposited; despite a sizeable root-mean squared (RMS) roughness of $3.4 \mathrm{~nm}$, no holes135 are present. After an annealing at $100^{\circ} \mathrm{C}(\mathrm{B})$, holes are still absent, surface coverage is still $100 \%$, but grains are larger and the roughness increased up to $5.2 \mathrm{~nm}$ RMS. This cor-

100 responds to the previously observed induction time [1, 7]. Holes appeared in the sample annealed at $150^{\circ} \mathrm{C}(\mathrm{C})$ at140 which the surface coverage dropped to $72 \%$. The silver domains are still interconnected but hillocks (i.e. grown grains) are now visible. At this stage, which is a typical 105 picture of hole propagation, the silver layer morphology can be divided into three categories: holes, hillocks and ${ }^{145}$ unchanged layer. After annealing at $300^{\circ} \mathrm{C}(\mathrm{D})$, the silver domains becomes tortuous or worm-like and the surface coverage is lowered $(48 \%)$; there is no remains of the initial layer: all silver domains have an increased thickness (at least $40 \mathrm{~nm}$ thick). This step corresponds to the begin-150 ning of sintering. After annealing at $400^{\circ} \mathrm{C}$, disconnected worm-like particles cover $39 \%$ of the surface. The layer consisting of objects of more regular shape is still evolving through sintering as the expected particle equilibrium shape is not yet reached.

To correlate the optical response with those observations, the dielectric function was extracted from post mortem ellipsometry considering an isotropic layer treated as a BSpline with a thickness fixed at $20 \mathrm{~nm}$ on a silicon sub-160 strate covered with its $2 \mathrm{~nm}$ thick oxide layer. Since bulk interband transitions in silver appear above $3.9 \mathrm{eV}$ [16], analysis will be focussed on the low energy range where
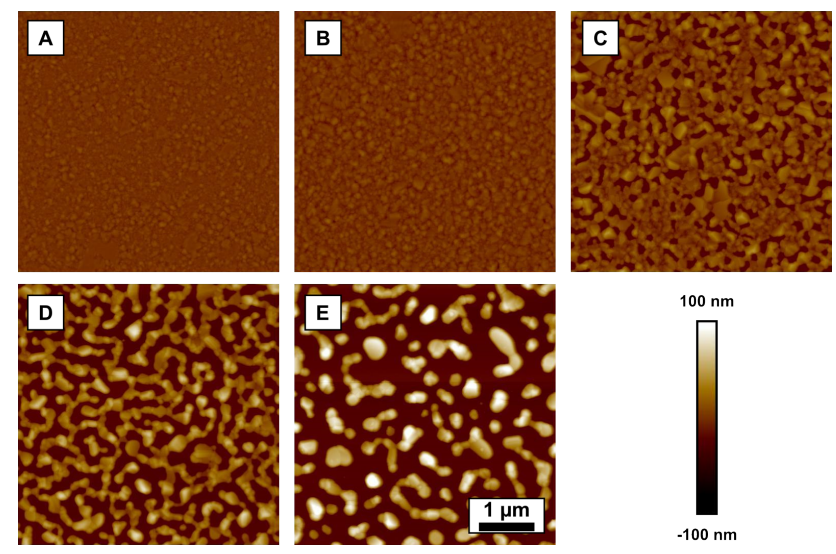

Figure 1: AFM topography characterization of a silver layer with a $20 \mathrm{~nm}$ initial thickness: a) as deposited, and after annealing at increasing temperatures b) $100^{\circ} \mathrm{C}$, c) $150^{\circ} \mathrm{C}$, d) $300^{\circ} \mathrm{C}$, e) $400^{\circ} \mathrm{C}$.

morphology changes show up (see Fig. 2).

Annealing at $100^{\circ} \mathrm{C}$ poorly impacts the dielectric function. Only grain growth and an increase of roughness were observed at this stage on Fig. 1. It is expected to induce a change in the conductivity properties through electron relaxation time, but this is hardly detectable in the probed spectral range. Working in near or mid infrared may be helpful in this regard [15], but it was not possible with the apparatus used in our study. The first steps of dewetting could be investigated in this range in future studies. However, consecutively to annealing at higher temperatures (above $150^{\circ} \mathrm{C}$ ), the real part of the dielectric function dramatically increases in the near-infrared range. The sample annealed at $300^{\circ} \mathrm{C}$ marks the transition between negative values obtained at lower temperatures and positive values obtained at higher temperatures. This transition corresponds to a shift between a metallic state and an insulating one. In parallel, the imaginary part of the dielectric function increases first in the near-infrared range, but decreases after annealing at $400^{\circ} \mathrm{C}$ while a complex shape emerges between 1.5 and $3.0 \mathrm{eV}$. This evolution of $\varepsilon$ is likely due to a transformation from a Drude-like behaviour to a oscillator-like (or Lorentzian) component due to the existence of large particles (which in-plane sizes can reach $1000 \mathrm{~nm}$ ). The peak between 1.5 and $3.5 \mathrm{eV}$ presumably comes from the coexistence of randomly oriented particles which sizes range between 150 and $250 \mathrm{~nm}$. Due to the dispersion of configurations, sizes, shapes and interactions, the signal is expected to have a Gaussian profile [13, 17]. As underlined before, it is difficult to study the early stages of propagation, because the changes in the ellipsometric spectra are small. However, hole percolation, which marks the beginning of sintering [7, can be easily detected (see below). It is correlated with the appearance of a signal between 1.5 and $3.0 \mathrm{eV}$ which parallels the formation of big and randomly oriented particles.

In situ experiments were performed on two different samples which led to two noticeably different final states; 


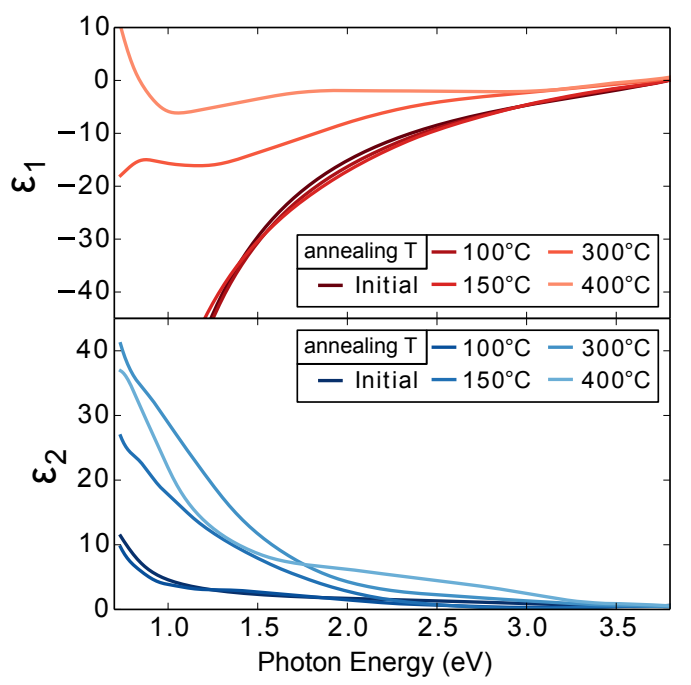

Figure 2: Dielectric function extracted from the ellipsometry analysis of post-mortem samples: as deposited or after annealing at 100, 150, 300 and $400^{\circ} \mathrm{C}$. Top: real part $\varepsilon_{1}$, bottom: imaginary part $\varepsilon_{2}$
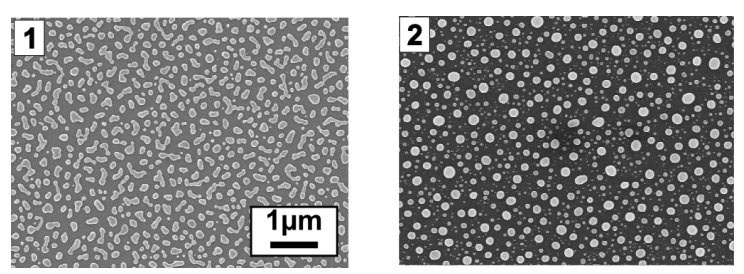

Figure 3: SEM observation of the final morphology of sample 1 and 2 analyzed by in situ ellipsometry after thermal treatment.

the first one consists of worm-like particles and the second 190 of regular round shaped particles as shown by SEM imaging (see Fig. 3). Theses differences of final state between samples from the same batch are difficult to explain: two samples from the same wafer and deposited at the same time can still differ in the way they dewet. Nonetheless we195 observe regularly the two types of dewetting. The question is then to know whether the difference is only due to kinetics, or if the evolution path is really different.

We first focus on the analysis of sample 1, because it requires a special treatment. Based on the post mortem ${ }^{200}$ findings, the dielectric function of the layer was assumed to be isotropic and was parametrized with three components: (i) a Tauc-Lorentz component describing the interband transitions above $3.9 \mathrm{eV}$; (ii) a Gaussian to account for the observed changes between 1.5 and $3.0 \mathrm{eV} ; 205$ (iii) a Lorentz component mimicking the Drude behaviour at early stages and marking the transition to an insulating layer. The attempts to add an actual Drude model led to fit instabilities due to the large number of degrees of freedom in the analysis. The Lorentz peak turned out to be ${ }^{210}$ more convenient in the energy range above $0.75 \mathrm{eV}$ to fit the measurements. In early stage of dewetting, it has an extremely high amplitude and is shifted to lower energies,
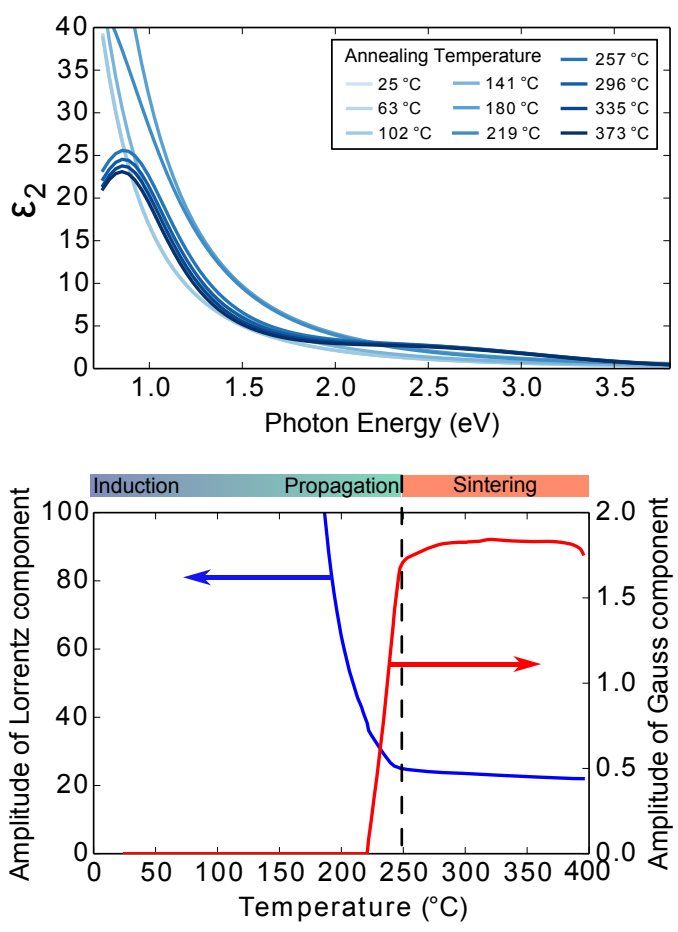

Figure 4: (Top panel): Imaginary part of the parametrized dielectric function of sample 1 measured in situ (Bottom panel): Amplitude of the Lorrentz component and the Gaussian component of the dielectric function (see text). The different stages of dewetting are indicated on top of the figure.

thus mimicking a Drude behaviour.

The fitted dielectric function is plotted at different times and therefore temperatures in Fig. 4. Firstly, $\varepsilon_{2}$ increases in the near-infrared before reaching a maximum at a temperature around $200^{\circ} \mathrm{C}$. Then $\varepsilon_{2}$ decays while the Gaussian component centred at $2.5 \mathrm{eV}$ appears. This behaviour is very similar to the dielectric function obtained with post mortem results. The parametrization allows studying the temperature evolution of the different components, in particular the amplitudes of the Lorentzian and Gaussian components as shown in Fig. 4. The amplitude of the Lorentzian peak that mimics the Drude behaviour is maximum up to $150^{\circ} \mathrm{C}$ before going through a sharp transition between 150 and $250^{\circ} \mathrm{C}$, after which it reaches its final value. Meanwhile, the Gaussian component rises quickly between 225 and $250^{\circ} \mathrm{C}$. After reaching this temperature, the evolution is much slower, corresponding to the slow kinetics of sintering. The Gaussian component can be assigned to the appearance of randomly oriented particles with sizes above $100 \mathrm{~nm}$. It is worth noticing that (i) the signal for disconnected particles (Gaussian component) appears later than the transition between the metallic state and the insulating state, meaning that they do not form directly during hole propagation (ii) the signal appears before the sintering, meaning that some particles are already formed in the early stages of sintering. It is then possible to classify particles into two distinct populations: first, the 


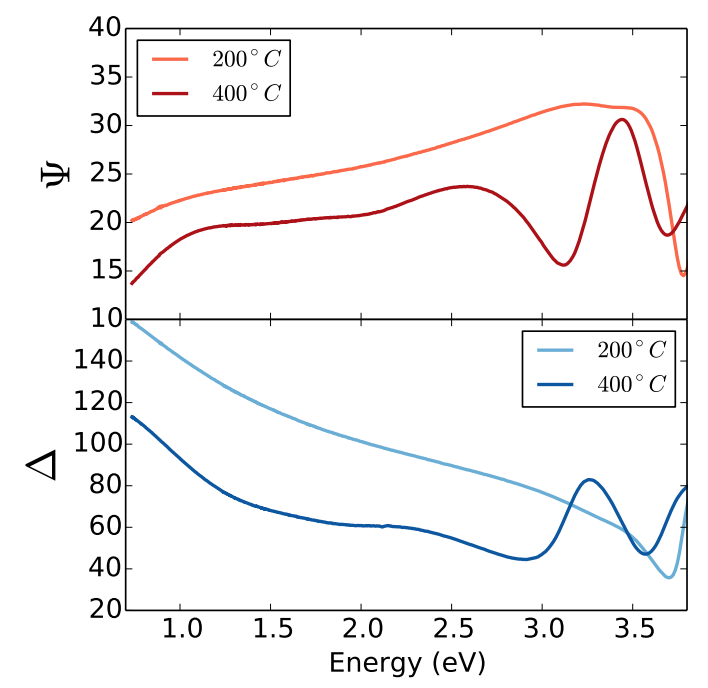

Figure 5: The $\Psi$ and $\Delta$ parameters recorded from sample 2 at 200 and $400^{\circ} \mathrm{C}$.

particles that have a shape far from the equilibrium, and that will require sintering to reach it, and then particles that reached it very quickly, at the end of propagation.

Although it is difficult to predict the time required to reach the thermal equilibrium shape from one sample to the other, the prompt formation of the desired particles was actually observed on sample 2 (see Fig. 3 right). Fig. 5 shows the extracted $\Psi$ and $\Delta$ parameters from sample 2 at 200 and $400^{\circ} \mathrm{C}$. A sharp peak around $3.2 \mathrm{eV}$ due to a localized plasmon resonance is clearly present after annealing at $400^{\circ} \mathrm{C}$, as well as a resonance around $2.5 \mathrm{eV}$. To study the evolution of this sample, an uniaxial model improved drastically the results of optimization. Therefore two B-splines were fitted, one along the z-axis normal to the surface and the other along the $\mathrm{x}$-axis parallel to it. $\mathrm{No}_{270}$ sizeable changes were observed on the out-of-plane component of the dielectric tensor. Therefore, on Fig. 6, only the imaginary part of the in-plane component of the dielectric tensor is plotted. At a temperature close to $300^{\circ} \mathrm{C}$, a sharp peak appears around $3.2 \mathrm{eV}$ while another less pronounced peak shows up at $2.5 \mathrm{eV}$. In parallel, the values for energies below $2.0 \mathrm{eV}$ decrease almost to zero.

This last observation is coherent with the disappearance of large worm-like particles. The particles of sample 2 observed in SEM can be divided into two distinct populations of sizes; the population of smaller sizes is centred around a value of $80 \mathrm{~nm}$ (in diameter) and the other one around $200 \mathrm{~nm}$. The two peaks observed in $\varepsilon_{2}$ may be related to these latter. Regarding the first population, its optical response can be modelled as truncated sphere on ${ }^{285}$ silicon in the framework of the quasistatic approximation using the GranFilm [18] code; their optical behaviour is mainly dominated by absorption. Preliminary simulations suggested that a parallel resonance should occur around ${ }^{290}$

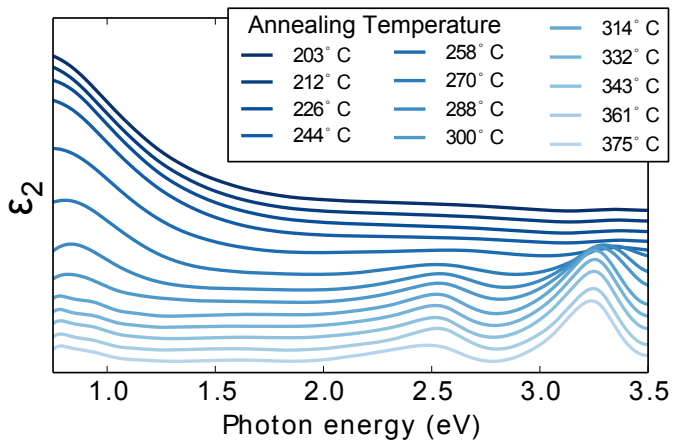

Figure 6: Evolution of the in-plane imaginary part of the dielectric function extracted during the annealing up to $400^{\circ} \mathrm{C}$ of sample 2

$3.2 \mathrm{eV}$, a value close to the present observations. According to this assignment, since the $3.2 \mathrm{eV}$ resonance appears at a temperature of $280^{\circ} \mathrm{C}$, it means that particles form at this temperature. Concerning larger particles, a resonance at lower energies is expected, but further work is required to account for multipolar scattering by those objects which size is a non negligible fraction of the wavelength. Nonetheless, we notice that the $2.5 \mathrm{eV}$ peak appears at the same temperature as the peak at $3.2 \mathrm{eV}$ and is probably linked to the second population of particles. This means that the equilibrium state observed on sample 2 is reached at a temperature below $400^{\circ} \mathrm{C}$, and that both populations appear at the same time, implying that the difference observed with sample 1 is not only a kinetic issue.

In conclusion, the potentiality of ellipsometry was explored in order to monitor in situ and in real time silver solid-state dewetting. Post mortem analysis of samples annealed at different temperatures allowed to link the main features in the dielectric function to the morphology of the film. Even though the early stages of dewetting (induction and beginning of propagation) may require a dedicated study at lower energies, it was possible to determine the transition between a metallic and an insulating state at $200^{\circ} \mathrm{C}$. The worm-like particles observed at the beginning of the sintering step have a specific signature; they appear only after the film breakup and further agglomeration $\left(\mathrm{T}=225^{\circ} \mathrm{C}\right)$, not at the beginning of the propagation step. The appearance at higher temperature $\left(\mathrm{T}=275^{\circ} \mathrm{C}\right)$ of round equilibrium-shaped particles that exhibit a specific signal was also captured.

The authors gratefully thank Ingve Simonsen (NTNU and SVI) for fruitful discussions.

[1] A.E.B. Presland, G.L. Price, and D.L. Trimm. Hillock formation by surface diffusion on thin silver films. Surf. Sci., 29(2):424434, 1972 .

[2] C. V. Thompson. Solid State Dewetting of Thin Films. Annu. Rev. Matter., 42:399, 2012.

[3] H. Atwater and A. Polman. Plasmonics for improved photovoltaic devices. Nature mat., 9(3):205-213, 2010. 
[4] S. Morawiec, M. Mendes, S. Mirabella, F. Simone, F. Priolo, and I. Crupi. Self-assembled silver nanoparticles for plasmonenhanced solar cell back reflectors: correlation between structural and optical properties. Nanotech., 24(26):265601, 2013.

[5] F. Niekiel, P. Schweizer, S. M. Kraschewski, B. Butz, and E. Spiecker. The process of solid-state dewetting of Au thin films studied by in situ scanning transmission electron microscopy. Acta Mater., 90:118-132, 2015.

[6] F. Niekiel, S. M. Kraschewski, P. Schweizer, B. Butz, and E. Spiecker. Texture evolution and microstructural changes during solid-state dewetting: A correlative study by complementary in situ TEM techniques. Acta Mater., 115(2016):230-241, 2016.

[7] P. Jacquet, R. Podor, J. Ravaux, J. Teisseire, I. Gozhyk, J. Jupille, and R. Lazzari. Grain growth: The key to understand solid-state dewetting of silver thin films. Scripta Mater., 115:128-132, 2016.

[8] S. A. Maier. Plasmonics: Fundamentals and applications. 2007.

[9] T. W. H. Oates and A. Mücklich. Evolution of plasmon resonances during plasma deposition of silver nanoparticles. Nanotech., 16(11):2606-2611, 2005.

[10] T. W. H. Oates, M. Losurdo, S. Noda, and K. Hinrichs. The effect of atmospheric tarnishing on the optical and structural properties of silver nanoparticles. J. Phys. D: App. Phys., 46(14):145308, 2013.

[11] T.W.H. Oates, H. Wormeester, and H. Arwin. Characterization of plasmonic effects in thin films and metamaterials using spectroscopic ellipsometry. Prog. Surf. Sci., 86(11-12):328-376, 2011.

[12] S. Grachev, M. DeGrazia, E. Barthel, E. Søndergård, and R. Lazzari. Real-time monitoring of nanoparticle film growth at high deposition rate with optical spectroscopy of plasmon resonances. J. Phys. D: App. Phys., 46(37):375305, 2013.

[13] R. Lazzari and J. Jupille. Quantitative analysis of nanoparticle growth through plasmonics. Nanotech., 22(44):445703, 2011.

[14] R. Lazzari and J. Jupille. Growth kinetics and size-dependent wetting of $\mathrm{Ag} / \alpha-\mathrm{Al}_{2} \mathrm{O}_{3}$ (0001) nanoparticles studied via the plasmonic response. Nanotech., 23(13):135707, 2012.

[15] F. Meng and A. Pucci. Growth of silver on $\mathrm{MgO}(001)$ and infrared optical properties. Phys. Status Solidi (b), 244(10):37393749, 2007.

[16] P. B. Johnson and R. W. Christy. Optical constants of the noble metals. Phys. Rev. B, 6:4370-4379, 1972.

[17] Y. Battie, A. EnNaciri, W. Chamorro, and D. Horwat. Generalized Effective Medium Theory to Extract the Optical Properties of Two-Dimensional Nonspherical Metallic Nanoparticle Layers. J. Phys. Chem. C, 118:4899-4905, 2014.

[18] R. Lazzari and I. Simonsen. Granfilm: a software for calculating thin-layer dielectric properties and fresnel coefficients. Thin Solid Films, 419:124, 2002. 